\title{
Die Natriumkanalblockade mit Phenytoin schützt den entzündeten Sehnerv
}

Fragestellung: Zeigt eine Natriumkanalblockade mit Phenytoin bei Patienten mit akuter Sehnerventzündnung neuroprotektive Wirkungen?

Hintergrund: Die akute Optikusneuritis ist ein häufiges Symptom der Multiplen Sklerose (MS) und kann aufgrund einer Neurodegeneration mit Faserverlust im Nervus opticus beziehungsweise der Retina zu persistierender Einschränkung der Sehschärfe führen. Die Hemmung spannungsabhängiger Natriumkanäle zeigt in experimentellen MS-Modellen neuroprotektive Effekte.

Patienten und Methodik: Es handelt sich um eine bizentrische, placebokontrollierte doppelblinde Phase-II-Studie aus England. Es wurden Patienten im Alter von 18 bis 60 Jahren mit einer

Raftopoulos R, Hickman SJ, Toosy A et al. Phenytoin for neuroprotection in patients with acute optic neuritis: a randomised, placebo-controlled, phase 2 trial. Lancet Neurol 2016; 15: 259-69 akuten Optikusneuritis und einem Visus von $\leq 0,66$ innerhalb von zwei Wochen nach Symptombeginn im Verhältnis $1: 1$ auf orales Phenytoin (4 bzw. $6 \mathrm{mg} / \mathrm{kg}$ Körpergewicht pro Tag) oder
Placebo randomisiert. Als primärer Endpunkt wurde die retinale Faserschichtdicke (retinal nerve fiber layer thickness, RNFLT) des betroffenen Auges nach sechs Monaten definiert, gemessen mithilfe der optischen Kohärenztomografie. Weitere untersuchte Parameter waren das Makulavolumen, die Läsionslänge, visuell evozierte Potenziale, die Sehschärfe und der Niederkontrastvisus.

Ergebnisse: Von 86 Studienteilnehmern erhielten 42 Phenytoin und 44 Placebo. Die mittere RNFLT nach sechs Monaten betrug $81,5 \mu \mathrm{m}$ in der Phenytoingruppe und $74,3 \mu \mathrm{m}$ in der Placebogruppe, was einer mittleren Abnahme der RNFLT innerhalb der Gruppen von 16,7 und 23,8 $\mu \mathrm{m}$ und damit einer Reduktion des Faserverlustes um rund $30 \%$ unter Phenytoin gegenüber Placebo entsprach. Während sich auch für das Makulavolumen ein positiver Behandlungseffekt ergab, zeigte sich in der Analyse der weiteren Parameter keine signifikante Wirksamkeit. Die Behandlung wurde insgesamt gut toleriert, ein Patient unter Phenytoin erlitt eine schwere Hautreaktion.

Schlussfolgerungen: Die Ergebnisse unterstützen die Hypothese, dass die Natriumkanalblockade mit Phenytoin bei Patienten mit akuter Optikusneuritis neuroprotektiv wirkt.

\section{- Kommentar von Ralf Linker, Erlangen}

\section{Erste Erfolge zur Neuroprotektion mit einem Natriumkanalblocker}

Die Studie ergibt erstmalig Hinweise für einen Axon schützenden Effekt durch eine Blockade spannungabhängiger Natriumkanäle bei entzündlicher Entmarkung im zentralen Nervensystem (ZNS) und stellt somit einen wichtigen Schritt auf dem Weg zur Entwicklung neuroprotektiver Therapien bei Erkrankungen wie der Multiplen Sklerose dar. Die höhere Nervenfaserdicke von knapp über $7 \mu \mathrm{m}$ unter Phenytoin ist zwar statistisch signifikant, aber absolut gesehen nur gering ausgeprägt. Ob diese Effektstärke auch klinische Relevanz haben kann, bleibt abzuwarten. Effekte auf den Visus konnten in der Arbeit nicht gezeigt werden, allerdings war die Studie hierfür auch nicht ausgelegt. Für die Anwendung im klinischen Alltag bleiben die Ergebnisse größerer, gut balancierter Phase-III-Studien abzuwarten, nachdem es in der vorliegenden Arbeit auch ein leichtes Ungleichgewicht zwischen den Studiengruppen hinsichtlich der mittleren Ausgangs-RNFLT sowie dem initialen Niederkontrastvisus gab. In jedem Falle legt die vorliegende Arbeit sehr schön nahe, dass ein enges Zeitfenster und eine rasche Eindosierung für den neuroprotektiven Behandlungserfolg mit Natriumkanalblockern entscheidend ist, nachdem die vorausgehende Studie mit Lamotrigin bei sekundär chronisch progredienter MS negativ blieb [1].
Auch für zukünftige Arbeiten ist die akute Optikusneuritis ein sehr gutes Paradigma, um neuroprotektive Effekte bei entzündlicher Entmarkung im ZNS zu testen [2]. Hier werden weitere Studien, eventuell auch mit noch besser verträglichen Natriumkanalblockern als Phenytoin, mit Spannung zu erwarten sein.
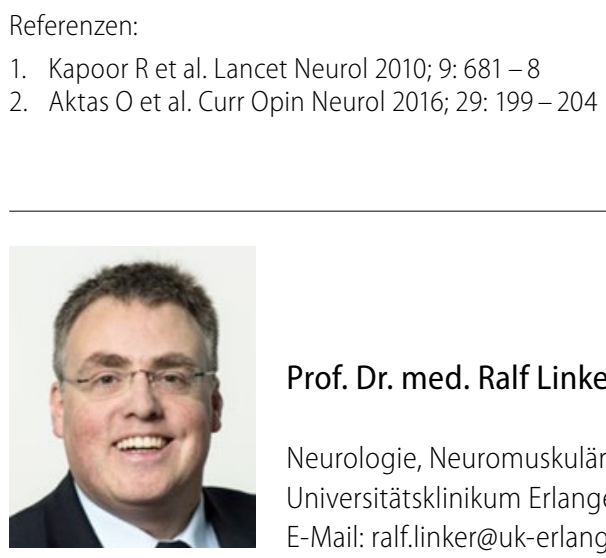

Prof. Dr. med. Ralf Linker, Erlangen

Neurologie, Neuromuskuläres Zentrum, Universitätsklinikum Erlangen E-Mail: ralf.linker@uk-erlangen.de 